cases had at least one traceable contact with confirmed attendance, and 122 had contacts testing positive. WGS confirmed links between traceable contacts in 82 cases. Reasons for unconfirmed links include contacts testing elsewhere and testing NAAT positive, culture negative. Untraceable contacts were reported in 157 cases; WGS provided possible links in 83, with confirmation in only six, given inherent information unavailability. Cases were grouped into 123 clusters, with eight containing $>10$ patients. Examination of clusters highlighted gaps in partner finding, including clusters containing heterosexual females with identical strains but no male; heterosexual males with identical strains who reported female sex worker contact; confirmed instances of partner underreporting; and 35 cases with multiple partners but no genetically related case.

Conclusion WGS has the potential to improve gonorrhoea PN and control by identifying new links and clusters with significant gaps in partner finding, where PN can be enhanced. Its utility will improve with larger databases.

Disclosure No significant relationships.

\section{O03.6 VAGINAL BACTERIA AND RISK OF INCIDENT AND PERSISTENT INFECTION WITH HIGH RISK SUB-TYPES OF HUMAN PAPILLOMAVIRUS}

${ }^{1}$ Kayla Carter*, ${ }^{2}$ Sujatha Srinivasan, ${ }^{3}$ Joshua Kimani, ${ }^{3}$ Omu Anzala, ${ }^{3}$ Emmanuel Kabare, ${ }^{3} J u m a$ Shafi, ${ }^{2}$ Elizabeth Brown, ${ }^{2}$ David Fredricks, ${ }^{1} \mathrm{R}$ Mcclelland, 'Jennifer Balkus. 'University of Washington, Department of Epidemiology, Seattle, USA; ${ }^{2}$ Fred Hutchinson Cancer Research Center, Vaccine and Infectious Disease Division, Seattle, USA; ${ }^{3}$ University of Nairobi, Nairobi, Kenya

\subsection{6/sextrans-2019-sti. 121}

Background Certain vaginal bacteria may increase women's risk for infection with high risk sub-types of human papilloma virus (hrHPV). The role of vaginal bacteria in hrHPV persistence is less well studied. We assessed associations between vaginal bacteria and hrHPV acquisition and persistence among Kenyan women in the placebo arm of the Preventing Vaginal Infections trial.

Methods Nonpregnant, HIV-uninfected women aged 18-45 from Kenya and the United States were enrolled in a randomized trial of periodic presumptive treatment to reduce vaginal infections over 12 months. Genital fluid specimens collected at enrollment and every 2 months thereafter were tested for 14 hrHPV types using the Hologic APTIMA HPV assay. Quantitative PCR targeting the 16S rRNA gene from ten bacteria was used to measure bacterial concentrations in vaginal swabs. Multivariate multinomial logistic regression and multistate Markov models restricted to Kenyan placebo participants were used to assess associations between $\log 10$-transformed bacterial concentrations (categorized in tertiles) and hrHPV acquisition and persistence.

Results Among the 84 Kenyan placebo participants, hrHPV was detected in $79 / 563$ specimens (14\%), with 16 episodes of persistent hrHPV (detection in $\geq 2$ consecutive specimens; median duration was 6 months). Controlling for age, hormonal contraceptive use, and condom use, Lactobacillus jensenii concentration was positively associated with hrHPV incidence (adjusted odds ratio $(\mathrm{aOR})=1.57$; 95\% CI 1.07-2.30); and Atopobium vaginae $(\mathrm{aOR}=1.40 ; 95 \%$ CI 1.02-1.93), Megasphaera species ( $\mathrm{aOR}=1.39 ; 95 \%$ CI 1.03-1.88), and Mageeibacillus indolicus $\quad(\mathrm{aOR}=1.48 ; \quad 95 \% \quad$ CI $1.09, \quad 2.02)$ concentrations were positively associated with hrHPV persistence. BV (Nugent score $\geq 7$ ) was not significantly associated with hrHPV incidence or persistence. Multistate Markov models did not indicate that bacterial concentrations were associated with transitions between HPV detection states.

Conclusion These findings suggest that higher concentrations of certain vaginal bacteria may increase risk of hrHPV incidence and persistence. Future work with more frequent sampling could provide additional insight into factors associated with hrHPV persistence.

Disclosure No significant relationships.

\section{4 - ANTIMICROBIAL RESISTANCE IN STI PATHOGENS}

Monday, July 15, 2019 4:15 PM - 5:45 PM

\begin{tabular}{l|l}
\hline 004.1 & NOVEL PATHWAY TO CEFTRIAXONE \\
RESISTANCE IN CLINICAL ISOLATES OF \\
N. GONORRHOEAE VIA POINT MUTATIONS \\
IN THE RNA POLYMERASE
\end{tabular}

Samantha Palace, Yi Wang, Daniel Rubin, Yonatan Grad*. Harvard T H Chan School of Public Health, Immunology and Infectious Diseases, Boston, USA

\subsection{6/sextrans-2019-sti.122}

Background Widespread antimicrobial resistance in Neisseria gonorrhoeae has limited the effective treatment options. Cephalosporins remain one of the few classes of antibiotics recommended for gonococcal infections, but reduced susceptibility to the third-generation cephalosporins, including ceftriaxone, has emerged. Most reduced susceptibility to ceftriaxone is caused by an alternative penA(PBP2) allele. However, the isolates with the among the highest level cephalosporin resistance identified in the US lack this allele and other penA resistance mutations, raising the possibility of cephalosporin resistance not mediated directly through penA.

Methods To identify the genetic basis of resistance in these isolates, we employed an undirected transformation strategy, and used molecular microbiology and genetics methods to investigate the mechanism of resistance.

Results Here, we show that resistance to ESCs has arisen in clinical isolates multiple times through distinct mutations in the RNA polymerase components $\mathrm{rpoB}$ and $\mathrm{rpoD}$. The resistance caused by these changes is not a general tolerance response: these mutations neither changed the growth rate in vitronor altered susceptibility to other classes of antibiotics (including penicillin). These mutations result in large variations in transcription, including in genes coding for penicillin binding proteins (increase in PBP1, decrease in PBPs 3 and 4) and pilus pore. We show that increases in PBP1 protein levels contribute to the rise in CRO MIC, likely through replacement of inhibited PBP2 activity, though other factors are needed to recapitulate the resistance seen in the clinical isolates with rpoB and rpoD mutations.

Conclusion Pathways to extended spectrum cephalosporin resistance do not require alterations to penA (PBP2) and can arise through mutations in components of the RNA polymerase holoenzyme. Additional pathways to cephalosporin 